\title{
D esafios na implantação de uma política de humanização da assistência hospitalar ao parto
}

\author{
Challenges for the implementation \\ of a humanization policy in hospital \\ care for childbirth
}

$M$ arcos Augusto Bastos Dias 1

Rosa M aria Soares M adeira Domingues 1

\footnotetext{
1 Secretaria M unicipal de Saúde do Rio de Janeiro. Rua Afonso Cavalcanti 445 sala 823, Cidade N ova 20211-901, Rio deJaneiro RJ. marcosad@centroin.com.br
}

A bstract The objectives of this essay are to argue the difficulties of implantation of a new model of assistance to low risk childbirth in the hospital structure and the systematization of a field of work to the obstetric nurse. From a new perspective of the care of the woman in labor, the authors make a reflection on the model of performance of this professional category, the possible positive re sults of this new assistance and of the conflict generated for the division of a space until then of exclusive domain of the medical category and the consequent shock of models of cares.

Key words Obstetric nurse, Humanization, Care, M odel of assistance, Childbirth
Resumo O s objetivos deste ensaio são discutir as dificuldades de implantação de um novo modelo de assi stência ao parto de baixo risco na estrutura hospitalar e na sistematização de um campo de trabalho da enfermeira obstetra. Sob uma nova perspectiva do cuidado da parturiente, os autores fazem uma reflexão sobre o modelo de atuação dessa categoria profissional, dos possíveis resultados positivos decorrentes dessa nova assistência e do conflito gerado pela divisão de um espaço até então de domínio exclusivo da categoria médica e do conseqüente choque de modelos de cuidados. Palavras-chave Enfermagem obstétrica, Humanização, Cuidado, M odelo de assistência, Parto 


\section{Introdução}

A noção de humanização vem sendo utilizada há vários anos, em especial na área da saúde, quando se fala em humanização da assistência. No campo da assistência ao parto, as discussões sobre a humanização trazem demandas antigas e, nos últimos anos, vários autores e organizações não-governamentais têm demonstrado suas preocupações com a medicalização excessiva do parto (Green et al., 1998; ReH uNa, 1993; Wagner, 1994), propondo modificações no modelo de assistência ao parto, principalmente naqueles de baixo risco. A Organização M undial de Saúde, desde o início da década de 1980 (OM S, 1985; 1996), tem trazido contribuições importantes para este debate ao propor o uso adequado de tecnologias para o parto e nascimento, com base em evidências científicas que contestam práticas preconizadas no modelo médico de atenção.

$\mathrm{N}$ a tentativa de modificar este modelo no município do Rio de Janeiro, foi iniciada, na década de 1990, a implantação da Política de Humanização do Parto e N ascimento. U ma das estratégias adotadas foi a hierarquização da assistência ao parto, com a implantação da assistência aos partos de baixo risco por enfermeiras obstetras, seguindo 0 exemplo bem-sucedido de alguns países europeus, onde a assistência a esses partos é prestada por profissionais não médicos.

A implantação da atenção aos partos de baixo risco por enfermeiras obstetras está associada a mudanças nas práticas e rotinas institucionais, já que se encontra inserida num contexto de humanização do parto e nascimento. A entrada da enfermagem obstétrica neste espaço de assistência, hoje ocupado pel os médicos obstetras, tem provocado embates entre estas categorias, dificultando a implantação da política de humanização. Este artigo tem por objetivo discutir al gumas questões suscitadas por esta proposta assistencial.

\section{H umanização da assistência ao parto. Na prática, do que estamos falando?}

O conceito de humanização da assistência ao parto inclui vários aspectos. Alguns estão relacionados a uma mudança na cultura hospitalar, com a organização de uma assistência realmente voltada para as necessidades das mulheres e suas famílias. M odificações na estrutura física também são importantes, transformando o espaço hospitalar num ambiente mais acoIhedor e favorável à implantação de práticas humanizadoras da assistência.

Contudo, a humanização da assistência ao parto implica também e, principalmente, que a atuação do profissional respeite os aspectos de sua fisiologia, não intervenha desnecessariamente, reconheça os aspectos sociais e culturais do parto e nascimento, e ofereça o necessário suporte emocional à mulher e sua família, facilitando a formação dos laços afetivos familiares e o vínculo mãe-bebê. Outros aspectos se referem à autonomia da mulher durante todo o processo, com elaboração de um plano de parto que seja respeitado pelos profissionais que a assistirem; de ter um acompanhante de sua escolha; de serem informadas sobre todos os procedimentos a que serão submetidas; e de ter os seus direitos de cidadania respeitados.

Embora haja evidências científicas suficientes para que se realizem modificações no modelo médico tradicional de assistência ao parto, desmedicalizá-lo implica perda de poder. Abandonar rotinas que adéquam o trabalho de parto (TP) ao modo de funcionamento do hospital e adotar outras que privilegiam 0 acompanhamento de sua fisiologia seria perder o controle do processo da parturição e modificar as referências do papel do médico neste contexto da assistência.

Para alguns autores (Davis-Floyd, 1994; Wagner, 1994), a formação médica, centrada no modelo biologicista cada vez mais seduzido pela tecnologia, seria incapaz de proporcionar ao profissional médico os atributos necessários para a assistência ao parto desta forma. A visão do TP e do parto como eventos de risco ea necessidade de conformá-los ao processo de produção do hospital implicam quase sempre a tentativa de regulação e controle do parto pelos médicos, levando a inevitáveis interferências.

Em vários países da Europa, por exemplo, 0 trabalho do médico obstetra está voltado para a assistência às gestantes de risco (Wagner, 1994). Com uma formação voltada principalmente para as complicações da gestação e do parto, a maioria dos médicos obstetras encara os partos como situações de risco e de necessário acompanhamento médico (Dias, 2001). 0 uso intensivo de tecnologia com conseqüentes intervenções faz parte da necessidade de oferecer segurança, que obstetras, em geral, não acreditam que o corpo da mulher possa oferecer. Estas constatações não excluem o médico 
obstetra do processo de humanização da assistência, mas apontam os desafios que estes profissionais precisam superar no sentido de modificar sua rotina de assistência aos partos de baixo risco atuando mais como cuidadores do que efetivamente como "especialistas" em patologia obstétrica.

Por outro lado, a formação profissional do enfermeiro obstetra, mais voltado para o cuidar, tem sido considerada aquela que possibilita ao profissional uma abordagem diferenciada na condução do TP (Wagner, 1994). Desde 1994, o currículo mínimo de enfermagem foi modificado e, atualmente, o único curso previsto para formação específica de profissionais não-médicos na área obstétrica consiste na especialização em enfermagem, nível de pós-graduação lato sensu. A formação da enfermeira obstétrica dá maior ênfase aos aspectos fisiológicos, emocionais e socioculturais do processo reprodutivo, privilegiando uma atuação fundamentada na compreensão do fenômeno da reprodução como singular, contínuo e saudável, no qual a mulher é o foco central, e que se desenvolve em um determinado contexto sóciohistórico (Riesco, 2002).

Vários trabalhos mostram que, quando acompanhadas por essas profissionais, as muIheres necessitam de menos anal gésicos, ocorrem menos intervenções e os resultados finais são melhores que aqueles produzidos pela assistência médica convencional (H odnett, 1989; Blanchette, 1995).

A atuação do enfermeiro na assistência ao parto normal ganha força em nosso meio a partir da discussão dos resultados do modelo médico de atenção e do exemplo da atuação das enfermeiras obstetras em países europeus, como Inglaterra e Holanda. $\mathrm{N}$ esses locais, $\mathrm{O}$ modelo de atenção ao parto inclui a importante atuação dessas profissionais, apresenta resultados perinatais muito bons e alta satisfação das usuárias com a assistência prestada (W iegers, 1996, Turnbull, 1996).

No Brasil, em 1998, o M inistério da Saúde (MS, 1998) reconheceu oficialmente a assistência ao parto por enfermeiro obstetra nos hospitais conveniados com o SUS e normalizou a remuneração desses profissionais. Como marco definidor de uma nova política de atenção ao parto, o M S propôs em 1999 a criação dos Centros de Parto Normal (CPN), unidades que permitem a assistência aos partos de baixo risco fora dos hospitais, com possibilidade de funcionamento sem médicos, ficando enfermeiros obstetras responsáveis por todos os cuidados prestados às mulheres e bebês.

Ainda nas políticas públicas na área de saúde da mulher, em 2001 o M inistério da Saúde editou o manual Parto, A borto ePuerpério - Assistência H umanizada à M ulher (MS, 2001). A publicação define o que considera humanização da assistência e defende uma nova perspectiva em que os cuidados prestados devem ser efetivamente benéficos, as intervenções desnecessárias devem ser evitadas e a privacidade e a autonomia materna preservadas.

A simples substituição do médico pela enfermeira obstetra não resulta necessariamente na humanização da assistência. $M$ as acreditamos que se a atuação desta profissional estiver inserida em uma nova proposta de cuidado, estão dadas as condições para a modificação do modelo de assistência.

\section{H umanização da maternidade pública e o choque de modelos no espaço do pré-parto - A experiência do município do Rio de Janeiro}

As maternidades públicas no Brasil geralmente atendem a maior parte das mulheres que não têm condições de consumir os serviços oferecidos pelas instituições privadas de saúde. A assistência ao parto nessas instituições é particularmente perversa porque anula de diversas formas a possibilidade de a mulher e de a família vivenciarem os aspectos subjetivos desse momento.

Muitas mulheres que procuram assistência na rede pública de serviços passam por uma rotina que se inicia com a busca de uma vaga, após uma provável peregrinação por al gumas unidades de saúde (Giffin et al., 2000), e que se completa com a separação da família na internação e com a permanência no pré-parto, usualmente um espaço coletivo, junto com outras mulheres também em trabal ho de parto ou com outras intercorrências obstétricas, sem qualquer privacidade ou atenção às suas necessidades particulares.

Desde 1994, a Secretaria M unicipal de Saúde do Rio de Janeiro (SM S/RJ) vem tentando mudar este quadro e propondo a implantação de uma política de qualificação da assistência perinatal que preconiza, entre outros pontos, a assistência ao parto de baixo risco por enfermeiras obstetras. Em 1990, uma maternidade municipal já havia implantado a assistência ao 
trabalho de parto e parto por enfermeiras obstétricas, obtendo bons resultados perinatais, porém sem modificações importantes no modelo de atenção. Em 1994, com a inauguração da maternidade Leila Diniz, a enfermagem obstétrica passou a atuar nessa unidade, seguindo os preceitos da humanização. E em 1998, a SM S/ RJ expandiu o trabalho da enfermagem obstétrica na assistência pré-natal eao parto para todos os serviços municipais da denominada Área Programática 3.3 (AP 3.3), região da cidade com maior volume anual de nascimentos, tendo por referencial a mudança de modelo de atenção e a ênfase na humanização da assistência.

Em nossa experiência temos percebido que de uma forma geral o pré-parto é um local de difícil permanência para os profissionais de saúde se a dor do trabalho de parto é percebida como um "sofrimento" contra o qual pouco se pode fazer. A perversidade do modelo, que não oferece o suporte emocional e medicaliza em excesso a assistência ao parto, está no fato de que grande parte das rotinas adotadas aumenta a intensidade do processo doloroso e pouco ou nada é oferecido para o seu alívio. Para muitos profissionais, portanto, permanecer continuadamenteno pré parto ao lado de mulheres queixando-se de dores, que não conseguem evitar, torna-se um sacrifício.

0 grande desafio que se coloca, para todos os profissionais que prestam esta assistência, é o de minimizar o sofrimento das parturientes, tornando a vivência do TP e parto em experiências de crescimento e realização para a muIher e sua família. Acreditamos em uma nova abordagem que estimule a participação ativa da mulher e seu acompanhante, que priorize a presença constante do profissional junto da parturiente, que preconize o suporte físico e emocional e o uso de novas técnicas de cuidado que permitam o alívio da dor, como o estímulo à deambulação e à mudança de posição, uso da água para relaxamento e massagens.

As enfermeiras obstetras têm sido seduzidas a se incorporar a este projeto, não só pela valorização de sua atuação profissional, mas também pela possibilidade do que Pires (1996) chamou de "assistência integral de enfermagem". Este modelo de assistência rompe com a fragmentação dos cuidados e permite que a profissional recupere a compreensão do processo de assistência, superando o parcelamento de tarefas que caracteriza o sistema tradicional de atenção.

U ma dificuldade inicial para a implantação do projeto era a de que, em um primeiro mo- mento, não havia disponível no mercado de trabal ho enfermeiras obstetras em número suficiente para que se pusesse em prática uma política de atuação destas profissionais na assistência ao parto de baixo risco. Com o apoio do M inistério da Saúde, a partir de 1998, foi possível, em pouco tempo, para várias escolas de enfermagem, realizar em todo o país 55 cursos de especialização em obstetrícia, que capacitaram 854 profissionais para a rede SUS (MS, 2001), muitas delas funcionárias da própria rede da SM S/RJ.

U ma outra dificuldade importante foi a reação dos médicos à presença das enfermeiras obstetras diretamente na assistência ao parto, resultando no pronunciamento do Conselho Regional de M edicina do Rio de Janeiro (Cremerj) sobre a questão, com a publicação de uma resolução que colocava sob responsabilidade do profissional médico toda a assistência praticada dentro do hospital, inclusive aquela praticada por enfermeira na assistência ao parto de baixo risco. Na mesma publicação, colocava também a obrigatoriedade da informação aos usuários do serviço de saúde de que na instituição os partos eram assistidos tanto por médicos quanto por enfermeiros. Estas medidas têm o objetivo claro de, em última instância, pressionar o médico para que ele não abra espaço na assistência ao parto de baixo risco para a enfermeira obstetra.

Assim, a introdução do enfermeiro obstetra na assistência ao parto de baixo risco tem tornado imperiosa uma negociação com os médicos obstetras. Se por um lado as enfermeiras poderiam aliviar a carga de trabalho da equipe médica, por outro tinham a possibilidade de estar pondo em prática seus conhecimentos e ganhando espaço e poder neste campo de atuação. Para poder enfrentar esta disputa, a assistência ao parto de baixo risco por enfermeiros obstetras tem sido apresentada como uma diretriz de política de saúde na assistência perinatal.

A presença do médico obstetra no pré-parto, atuando em parceria com o enfermeiro obstetra na equipe, tem sido o modelo proposto para as maternidades da SM S/RJ. 0 trabal ho conjunto permitiria ao mesmo tempo uma atenção menos intervencionista, nos casos de baixo risco cuidados pelas enfermeiras, e uma atenção mais direcionada dos médicos obstetras aos casos de risco. Este modelo prevê que a atuação dos diversos profissionais deva ser complementar e que deve haver respeito aos limites de atuação de cada categoria. Entretanto, 
se isso não ocorre, frustram-se as expectativas profissionais e surgem conflitos entre os membros da equipe. 0 argumento de que com a presença da enfermeira no pré-parto o profissional médico pode se ocupar principalmente das pacientes com algum tipo de risco, realizar cirurgias e permanecer na sala de admissão onde são atendidas as emergências, é utilizado nas negociações que se estabelecem entre a equipe médica e de enfermeiras obstetras, para que estas tenham autonomia de atuação na condução da assistência às gestantes sem risco.

Como a assistência prestada pelos enfermeiros propõe-se ideal mente a acontecer em um modelo de atenção voltado para o cuidado, um enfrentamento com as condutas mais intervencionistas adotadas pelos médicos tornase praticamente inevitável. A utilização de recursos alternativos para a condução do trabaIho de parto como as bolas de fisioterapia, massagens, banhos de chuveiro ou banheira e mesmo a adoção de posições como a de cócoras durante $o$ trabalho de parto, foram encaradas inicialmente pelos médicos como pouco científicas e inapropriadas. Também a assistência ao parto em posições verticalizadas, diferentes das usualmente utilizadas pelos médicos, foi motivo de estranhamento entre os profissionais. Os médicos, preocupados com processos éticose judiciais, manifestaram suas preocupações em assumir os casos complicados que estivessem sendo conduzidos por enfermeiras.

Em nossa experiência na $M$ aternidade Leila Diniz, observamos que nos primeiros meses de consolidação da assistência ao parto pelas enfermeiras obstetras, a negociação com o grupo de médicos se fazia pela convivência no préparto, com a "supervisão" dos médicos e sua freqüente intervenção, nem sempre de acordo com 0 desejo das enfermeiras obstetras. $M$ as foi possível para a maioria dos médicos, ao passar dos meses, ganhar confiança no trabal ho das enfermei ras e avaliar a eficácia de algumas práticas, como a adoção de posições verticais durante o TP e parto e a utilização de técnicas não farmacológicas de alívio da dor, como as citadas anteriormente.

Como estratégia de facilitação de implantação desta política têm sido realizados encontros com o corpo clínico das maternidades. $\mathrm{N}$ as reuniões é discutida a política de humanização do parto e nascimento, e são realizadas oficinas de aproximação das equipes de médicos e enfermeiros obstetras, estabelecendo o trabalho em equipe como meta para a qualificação da assis- tência. O utra ação adotada é a realização de encontros da Câmara Técnica de Enfermagem Obstétrica para discutir as práticas de assistência e as estratégias adotadas nas diversas unidades para garantir sua autonomia na atenção.

\section{Considerações finais}

O modelo brasileiro de assistência ao parto e nascimento é em sua grande maioria extremamente intervencionista, tratando-os como eventos eminentemente médicos. Penaliza a mulher e sua família ao ignorar a fisiologia e os aspectos sociais e culturais do parto, tendo como resultado taxas de morbimortalidade materna e perinatal incompatíveis com os avanços tecnológicas ao nosso alcance. A proposta de humanização da assistência ao parto vem ao encontro de duas grandes demandas das mulheres e da sociedade. A primeira, a da oferta pelo sistema público de saúde de um serviço que não seja utilizado apenas por quem não tem qualquer outra opção, mas um serviço que devolva com qualidade às mulheres, o direito de parir em condições que assegurem sua segurança e a de seu bebê. A segunda que ofereça à mulher, na vivência deste acontecimento, a possibilidade de atuar como protagonista, fazendo com que o parto deixe de ser um evento meramente biológico, e sim uma experiência humana, que deve ser experimentada de acordo com suas expectativas, que podem estar demonstradas em um plano de parto previamente elaborado.

Embora não seja uma garantia de mudança do modelo de assistência ao parto, a inclusão da enfermeira obstetra na assistência ao parto de baixo risco tem mostrado que é medida capaz de reduzir as intervenções médicas desnecessárias e de oferecer um cuidado mais integral, dando o necessário suporte emocional à mulher e sua família. Ao mesmo tempo, é desejável que os médicos obstetras estejam redefinindo seu papel na assistência ao parto, seja por uma atenção menos intervencionista na assistência ao baixo risco, compreendendo a importância do suporte físico e emocional para a parturiente e estimulando sua adoção, seja por um maior foco na assistência às gestantes de risco e às urgências e emergências obstétricas. Trabalhando em equipe com a enfermagem obstétrica seria possível ao médico obstetra participar da assistência obstétrica de forma integral, atendendo desde as complicações surgidas na assistência à gestante de baixo risco 
durante o trabalho de parto, até a prestação da assistência às gestantes que já sabi damente necessitariam não apenas de acompanhamento, mas efetivamente de intervenções médicas de qualidade que pudessem garantir sua saúde e de seu bebê.

A decisão política de implantação da enfermeira obstetra na assistência ao parto de baixo risco necessita vir acompanhada de estratégias de apoio para a sua concretização em virtude da disputa inevitável pelo mercado da assistência obstétrica que esta medida acarreta. Os programas de ensino, treinamento e aperfeiçoamento precisariam oferecer aos enfermeiros e aos médicos obstetras o conhecimento não apenas dos aspectos médicos, mas também a sensibilização para os aspectos afetivos da gestação necessários a uma assistência adequada às gestantes. É necessário prover também aos médicos obstetras que atendem os casos de risco o treinamento para a execução das técnicas de operatória obstétrica, capaz de garantir a estes profissionais, maior segurança na condução dos partos vaginais com al gum tipo de complicação, evitando a indicação de cesarianas desnecessárias.

A proposta de humanização da assistência ao parto sofre influência direta do modelo organizacional, dos desenhos da missão institucional, do envolvimento e aderência dos gerentes à proposta, da capacitação e sensibilidade dos profissionais, mas a sua ef etiva implantação estará sempre atrelada à insubstituível relação entre a mulher e o profissional de saúde, uma relação entre dois seres humanos e, portanto, sujeita aos inevitáveis aspectos de suas subjetividades.

\section{Colaboradores}

MAB Dias e RM SM Domingues elaboraram de forma conjunta a redação do artigo de opinião.

\section{Agradecimentos}

À professora doutora Suely Deslandes pelo estímulo à produção do texto.

\section{Referências bibliográficas}

Barros FC, Vaughan JP, Victora CC \& Útil SR 1991. Epidemic of Cesarean sections in Brazil. Lancet 338:167169.

Blanchette H 1995. Comparison of obstetric outcome of a primary-care access clinic staffed by certified nursemidwives and a private practice group of obstetricians in the same community. Am J Obst. Gynecol 172(6):1864-1868.

Davis-Floyd RE1994. The rituals of American H ospital Birth, pp. 323-340. In D M cCurdy (ed.) Conformity and Conflict: Readings in Cultural Anthropology. Harper Collins, Nova York.

Dias M AB 2001. Cesariana: epidemia desnecessária? A construção da indicação da cesariana em uma maternidade pública do M unicípio do Rio de Janeiro. Dissertação de mestrado. IFF / Fiocruz, Rio de Janeiro.

Diniz SG \& Chacham A 2002. Humanização do parto dossiê. Rede Nacional Feminista de Saúde, Direitos Sexuais e Direitos Reprodutivos. (M imeo).

Giffin K, D'Orsi E, Reis AC, Barbosa GP et al. 2000. Fatores associados à realização de cesárea e qualidade da atenção ao parto em duas maternidades do Rio de Janeiro - sumário de resultados. Núcleo de Gênero e Saúde. ENSP-Fiocruz.

Green JM , Coupland VA \& Kitzinger JV 1988. Great expectations. A prospective study of women's expectations and experiences of childbirth. Books for Midwives Press, Inglaterra.

Hodnett ED \& Osborn RW 1989. A randomized trial of the effect of monitrice support during labor: mothers' views two to four weeks postpartum. Birth 16:177-183. 
M inistério da Saúde 2001. Programa Nacional de Humanização da Assistência H ospitalar - PNHAH. Disponível em <www.humaniza.org.br>

M inistério da Saúde 2001. Parto, aborto e puerpério: assistência humanizada à mulher. Brasília.

M inistério da Saúde 1998. Portaria no 2.816, de 29 de maio de 1998. Diário Oficial da República Federativa do Brasil no 103, 2 jun. Brasília.

OM S (Organização M undial de Saúde) 1985. Appropriate technology for birth. Lancet 24;2(8452):436-7.

OM S (Organização M undial de Saúde) 1996. Care in normal birth; a pratical guide. M aternal and N ewborn Health / Safe M otherhood Unit. Family and Reproductive H ealth. Genebra; W orld H ealth Organization.

Pires D 1996. Processo de trabalho em saúde no Brasil, no contexto das transformações atuais na esfera do trabaIho. Tese de doutorado. Instituto de Filosofia e Ciências H umanas, U niversidade Estadual de Campinas.

REH UNA (Rede Pela Humanização do Parto e do Nascimento) 1993. Carta de Campinas. (M imeo).
Riesco MLG \& Tsunechiro MA 2002. Midwifery and nurse-midwifery education: old problems or new possibilities? Rev. Estud. Fem. 10(2):449-459.

Turnbull D, H olmes A, Shields N, Cheyne H, TwaddleS, Gilmor WH et al. 1996. Randomised, controlled trial of efficacy of midewife-managed care. Lancet 348 (9022):213-218.

Wagner M 1994. Pursuing the birth machine. The search for appropriate birth technology. Camperdown, Austrália. ACE Graphics.

Wiegers TA, Keirse MJ, van der ZeeJ \& Birghs GA 1996. Outcome of planned home and planned birth in low risk pregnancies: prospective study in midwifery practices in The N etherlands. BM J nov 23; 313(7068): 1276-7

Artigo apresentado em 16/03/2005

Aprovado em 19/04/2005

Versão final apresentada em 3/05/2005 